The AstrophysicAL Journal, 506:253-258, 1998 October 10

(C) 1998. The American Astronomical Society. All rights reserved. Printed in U.S.A.

\title{
MICROLENS PARALLAX ASYMMETRIES TOWARD THE LARGE MAGELLANIC CLOUD
}

\author{
ANDREW GOULD ${ }^{1}$ \\ Ohio State University, Department of Astronomy, 174 West 18th Avenue, Columbus, OH 43210; gould@astronomy.ohio-state.edu \\ Received 1998 February 16; accepted 1998 May 7
}

\begin{abstract}
If the microlensing events now being detected toward the Large Magellanic Cloud (LMC) are due to lenses in the Milky Way halo, then the events should typically have asymmetries of order $1 \%$ owing to parallax from the reflex motion of the Earth. By contrast, if the lenses are in the LMC, the parallax effects should be negligible. A ground-based search for such parallax asymmetries would therefore clarify the location of the lenses. A modest effort ( $2 \mathrm{hr}$ per night on a $1 \mathrm{~m}$ telescope) could measure 15 parallax asymmetries over $5 \mathrm{yr}$ and therefore marginally discriminate between the halo and the LMC as the source of the lenses. A dedicated $1 \mathrm{~m}$ telescope would approximately double the number of measurements and would therefore clearly distinguish between the alternatives. Compared to satellite parallaxes, however, the information extracted from ground-based parallaxes is substantially less useful for understanding the nature of the halo lenses (if that is what they are). The backgrounds of asymmetries owing to binary-source and binary-lens events are estimated to be approximately $7 \%$ and $12 \%$, respectively. These complicate the interpretation of detected parallax asymmetries, but not critically.
\end{abstract}

Subject headings: dark matter — Galaxy: halo — gravitational lensing — Magellanic Clouds

\section{INTRODUCTION}

If the eight microlensing events observed by the MACHO collaboration toward the Large Magellanic Cloud (LMC) are due primarily to lenses in a standard Milky Way halo, then these lenses comprise about half of the halo mass within $50 \mathrm{kpc}$ and have typical masses $M \sim 0.4 M_{\odot}$ (Alcock et al. 1997a). Such objects could not be composed of hydrogen because they would burn and the population would thus be easily detected in star counts. The mass scale is roughly consistent with that of white dwarfs, but to date there are no plausible schemes for making enough white dwarfs to account for the microlensing events without also generating various easily observed effects such as the overproduction of metals.

A number of alternative suggestions have therefore been advanced for the origin of these puzzling events. Sahu (1994) and $\mathrm{Wu}$ (1994) proposed that the events are due to LMC lenses. Zhao (1998) suggested that they lie in a dwarf (possibly tidally disrupted) galaxy along the line of sight to the LMC, and Zartisky \& Lin (1997) claim to have detected such a structure. Evans et al. (1998) suggested that the lenses could lie in a warped and flared Milky Way disk. A variety of arguments have been advanced against these alternatives (Gould 1995a; Beaulieu \& Sackett 1998; Alcock et al. 1997a, 1997b; Gould 1998; Bennett 1998), and the nature of the events remains unresolved.

The fundamental problem is that microlensing events are generally described by a five-parameter light curve (Paczyński 1986),

$$
F\left(t ; t_{0}, \beta, t_{\mathrm{E}}, F_{0}, B\right)=F_{0} A\left[u\left(t ; t_{0}, \beta, t_{\mathrm{E}}\right)\right]+B,
$$

where $A(u)=\left(u^{2}+2\right) /\left[u\left(u^{2}+4\right)^{1 / 2}\right], u(t)$ is the projected separation of the source and lens in units of the Einstein radius, $r_{\mathrm{E}}$,

$$
u\left(t ; t_{0}, \beta, t_{\mathrm{E}}\right)=\left[\beta^{2}+\left(\frac{t-t_{0}}{t_{\mathrm{E}}}\right)^{2}\right]^{1 / 2},
$$

\footnotetext{
${ }^{1}$ Alfred P. Sloan Foundation Fellow.
}

$t_{0}$ is the time of closest approach, $\beta$ is the impact parameter in units of $r_{\mathrm{E}}, t_{\mathrm{E}}$ is the characteristic time of the event, $F_{0}$ is the unmagnified flux of the source star, and $B$ is any background light in the source aperture that is unaffected by the lensing event. Of the five parameters in equation (1), only $t_{\mathrm{E}}$ is in any way related to the physical characteristics of the lens. Moreover, this relation is rather indirect:

$$
t_{\mathrm{E}}=\frac{r_{\mathrm{E}}}{|v|},
$$

where

$$
r_{\mathrm{E}}=\left(\frac{4 G M d_{\mathrm{ol}} d_{\mathrm{1s}}}{c^{2} d_{\mathrm{os}}}\right)^{1 / 2}
$$

is the Einstein radius, $M$ is the mass of the lens, $d_{\mathrm{ol}}, d_{1 \mathrm{~s}}$, and $d_{\text {os }}$ are the distances between the observer, source, and lens, and $v$ is the transverse velocity of the lens relative to the observer-source line of sight. Thus, the experiment does not directly measure the mass, distance, or speed separately. The mass can be estimated only statistically and then only for given models of the distance and velocity distributions of the lenses and sources.

The most straightforward way to determine the location of the lenses would be to launch a parallax satellite into solar orbit (Refsdal 1966; Gould 1994, 1995b; Boutreux \& Gould 1996; Gaudi \& Gould 1997a; Marković 1998). Since the Earth-satellite separation $(\sim \mathrm{AU})$ is of order the size of the Einstein radius projected onto the plane of the observer, $\tilde{r}_{\mathrm{E}}$, the event parameters as seen from the Earth $\left(t_{0}, \beta, t_{\mathrm{E}}\right)$ will be substantially different from those seen by the satellite $\left(t_{0}^{\prime}, \beta^{\prime}, t_{\mathrm{E}}^{\prime}\right)$. From the difference one can infer the projected velocity $\tilde{v}$ or, equivalently, the projected Einstein radius, $\tilde{r}_{\mathrm{E}}$, and the direction of relative motion, $\phi$,

$$
\tilde{\boldsymbol{v}}=\frac{d_{\mathrm{os}}}{d_{\mathrm{ls}}} \boldsymbol{v}, \quad \tilde{r}_{\mathrm{E}}=\tilde{v} t_{\mathrm{E}} .
$$

For disk, halo, and LMC lenses, the projected velocities are typically 50,300 , and more than $1000 \mathrm{~km} \mathrm{~s}^{-1}$. Hence, parallaxes would allow one to identify the population to which 
the lenses belong on an almost case by case basis. Even lenses in the Evans et al. (1998) warped and flared disk could be distinguished from halo lenses (which have similar distances and speeds) because the former would be streaming in the direction of the disk while the latter would be approximately randomly distributed in $\phi$.

Since the launching of a satellite is a long-term and expensive undertaking, a number of other ideas have been explored for resolving the nature of the lenses. Binary lenses have a caustic in their magnification structures. If this caustic transits the source, then the proper motion of the lens, $\mu=\theta_{*} / t_{*}$, can be determined from the radius crossing time, $t_{*}$, and the angular radius of the source, $\theta_{*}$ (known from Stefan's law). The proper motion $\mu=v / d_{\mathrm{ol}}$ is more than 1 order of magnitude larger for halo lenses than for LMC lenses, so these two populations could be easily distinguished. In fact, the proper motion of one LMC event has been tentatively measured (Alcock et al. 1997a) and appears to be in the LMC. However, binary lenses may not be representative of the lens population as a whole. For example, it could be that binary lenses occur only in the LMC and that no halo lenses are binaries. Thus, no definitive conclusion can be drawn from measuring binary-lens proper motions. Proper motions can also be obtained for some binary-source events (Han \& Gould 1997). Unlike the binary-lens events, binary-source events are not intrinsically biased. They are, however, relatively rare and to date none have been unambiguously detected.

Another possible approach is ground-based parallaxes. The motion of the Earth causes the projected source-lens separation to deviate from the straight line reflected in equation (2) (Gould 1992; Gould, Miralda-Escudé, \& Bahcall 1994; Buchalter \& Kamionkowski 1997) and hence causes the light curve to deviate from equation (1). One measures the same parameters as with satellite parallaxes, $\tilde{v}$, or equivalently $\tilde{r}_{\mathrm{E}}$ and $\phi$. There is one published parallax for an event seen toward the Galactic bulge (Alcock et al. 1995) and several more that have not yet been published (Bennett et al. 1997). These events are typically very long, $t_{\mathrm{E}} \gtrsim 90$ days. The velocity of the Earth changes substantially during the course of a long event, and this is what allows the parallax to be measured. Most events seen toward the LMC are much shorter, $t_{\mathrm{E}} \sim 40$ days. Moreover, the LMC source stars are typically much fainter than the bulge sources, making it more difficult to acquire the accurate photometry required to detect the subtle parallax effect. However, the first event seen toward the Small Magellanic Cloud (SMC) (Alcock et al. 1997c; Palanque-Delabrouille et al. 1998) has $t_{\mathrm{E}} \sim 120$ days and has an exceptionally bright source $(V \sim 17)$. These characteristics allowed PalanqueDelabrouille et al. (1998) to put a lower limit on the projected speed $\tilde{v}>270 \mathrm{~km} \mathrm{~s}^{-1}$.

Gould et al. (1994) showed that even when the events are too short to allow one to make a complete parallax determination (and therefore to measure $\tilde{\boldsymbol{v}}$ ), parallax effects can nonetheless induce deviations of the light curves relative to equations (1) and (2). It might then be possible to detect the "parallax asymmetry" of some of these events. Measurement of this asymmetry yields the parameter combination $K_{\|}=\cos \phi / \tilde{v}$, where $\phi$ is the angle between $v$ and the EarthSun separation vector. Of course, it would be much better to measure $\tilde{v}$ and $\phi$ separately. In particular, if $K_{\|}$were found to be consistent with zero, one would not know whether $\tilde{v}$ was very large (indicating that the lens was almost certainly in the LMC) or $|\cos \phi|$ just happened to be very small. However, if $K_{\|}$were measured for a sample of events, one might be able to determine statistically whether most of the lenses were in the LMC (very few detections of $K_{\|}$) or whether many were in the Milky Way halo or disk (many detections of $K_{\|}$).

At the time that Gould et al. (1994) analyzed parallax asymmetries, the only event seen toward the LMC had a timescale $t_{\mathrm{E}} \sim 17$ days. If the lens were in the halo, the asymmetry in the light curve would be substantially smaller than $1 \%$, probably too small to detect reliably. Thus, Gould et al. (1994) considered parallax asymmetries primarily as a method to distinguish between halo lenses and disk lenses, the latter generating much larger asymmetries. T. Axelrod (1997, private communication), however, has pointed out that since the typical timescale of the observed events is now $t_{\mathrm{E}} \sim 40$ days, the asymmetries expected for halo lenses are typically much larger.

Here I elaborate on the work of Gould et al. (1994) to develop an analytic framework within which one can assess the detectability of parallax asymmetries. I also identify some backgrounds that may complicate the interpretation of any detections that are made.

\section{PARALLAX ASYMMETRIES}

In the limit of short events, the acceleration of the Earth may be regarded as constant over the duration of the event. Equation (1) then remains valid provided that equation (2) is replaced by (Gould et al. 1994)

$$
u(t)=\left\{\left[\xi\left(\frac{t-t_{0}}{t_{\mathrm{E}}}\right)\right]^{2}+\beta^{2}\right\}, \quad \xi(y)=y+\frac{1}{2} \gamma y^{2},
$$

where

$$
\gamma=\frac{v_{\oplus}}{\tilde{v}} \frac{t_{\mathrm{E}}}{\mathrm{yr} / 2 \pi} \cos \phi
$$

$v_{\oplus} \sim 30 \mathrm{~km} \mathrm{~s}^{-1}$ is the speed of the Earth, and where I have made use of the fact that the LMC is approximately at the ecliptic pole. Note that for typical observed LMC events $\left(t_{\mathrm{E}} \sim 40\right.$ days) and for projected velocities that are typical of the halo $\left(\tilde{v} \sim 275 \mathrm{~km} \mathrm{~s}^{-1}\right)$, the parameter $\gamma \sim 0.08 \cos \phi$ is small compared to unity. Also note that at $t_{\mathrm{E}} \sim 40$ days, the assumption that the Earth's acceleration vector is constant is beginning to break down; however, for purposes of estimating the detectability of parallax effects, this approximation is adequate.

Equation (6) introduces an asymmetry into the light curve. The lens gradually speeds up (or slows down) during the course of the event, so the rise time of the event is longer (or shorter) than the fall time. The first questions to address are the following: what is the maximal fractional deviation of the parallax-affected light curve from the unperturbed light curve $(\gamma=0)$, and at what time does this maximum deviation occur? I find

$$
(\Delta \ln F)_{\max }=\gamma W(\beta), \quad t_{\max }=t_{0} \pm \tau(\beta) t_{\mathrm{E}},
$$

where $W(\beta)$ is shown as a bold curve and $\tau(\beta)$ is shown as a solid curve in Figure 1. In making this evaluation, I have used equation (1) and have assumed $B / F_{0} \ll 1$. Obviously, in the case of significant blending, the maximum deviation will be smaller.

According to Figure 1, the maximum deviation occurs about 1 Einstein crossing time from the peak of the event, 


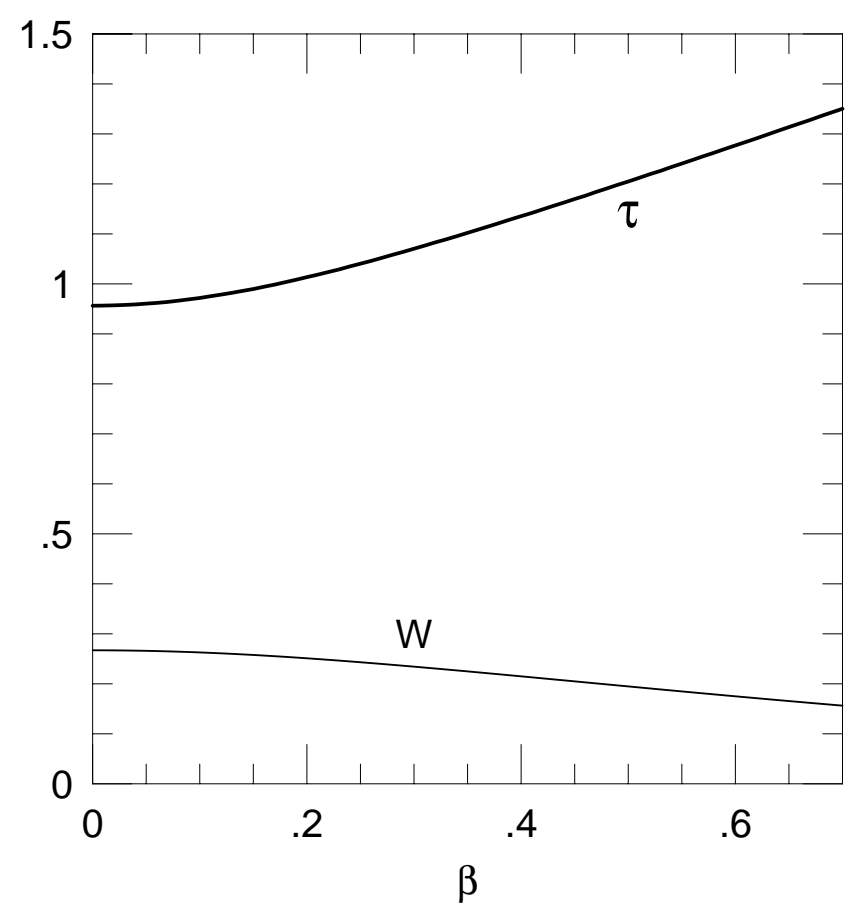

FIG. 1.-Asymmetry functions $W$ and $\tau$ plotted against the impact parameter $\beta$. The maximum fractional deviation of the light curve from its standard Paczyński (1986) form is given by $(\Delta \ln F)_{\max }=W \gamma$, where $\gamma$ is the asymmetry parameter defined by eq. (7). This maximum deviation occurs at times $t=t_{0} \pm \tau t_{\mathrm{E}}$.

more or less independent of the impact parameter. This will have importance in understanding the detectability of the events. Note that $W$ also depends only weakly on $\beta$. The typical value is $W \sim 0.2$. This implies that the maximum deviation for a typical halo event is $(\Delta \ln F)_{\max } \sim 0.2 \gamma \sim$ $1.5 \% \cos \phi$. This is certainly well below the photometric precision of the microlensing surveys that are searching for events. However, $1 \%$ photometry could be achieved by aggressive follow-up observations even for the typical sources that are faint $(V \sim 20)$ and in crowded fields.

The next question is then with what precision can $\gamma$ be measured assuming such a program is undertaken? Of course, $\gamma$ must be measured simultaneously with the other five parameters shown in equations (1) and (6). Employing standard techniques (see, e.g., Gould \& Welch 1996), I evaluate the covariance matrix $c_{i j}$ of the six parameters $a_{i}$ by considering a series of measurements at times $t_{k}$ and with errors $\sigma_{k}$,

$$
c=b^{-1}, \quad b_{i j}=\sum_{k} \sigma_{k}^{-2} \frac{\partial F\left(t_{k}\right)}{\partial a_{i}} \frac{\partial F\left(t_{k}\right)}{\partial a_{j}} .
$$

After taking the derivatives $\partial F\left(t_{k}\right) / \partial a_{i}$, I evaluate them assuming $B=\gamma=0$. I assume that the errors are $\sigma_{k}=$ $\sigma_{0} A\left(t_{k}\right)$. That is, they are limited by systematics, not statistics. I assume that these intensive observations are triggered when the event enters the Einstein ring $\left[u_{i}=1\right.$, $\left.A\left(u_{i}\right)=1.34\right]$ and end at $t=t_{0}+1.5 t_{\mathrm{E}}$, and that they are carried on uniformly at a rate $N$ per day in the interval. I then find an uncertainty in the determination of $\gamma$,

$$
\sigma_{\gamma}=\frac{\sigma_{0}}{\left(N t_{\mathrm{E}} \mathrm{day}^{-1}\right)^{1 / 2}} S(\beta),
$$

where $S(\beta)$ is shown as a bold curve in Figure 2. Combining this equation with equation (7) gives the signal-to-noise

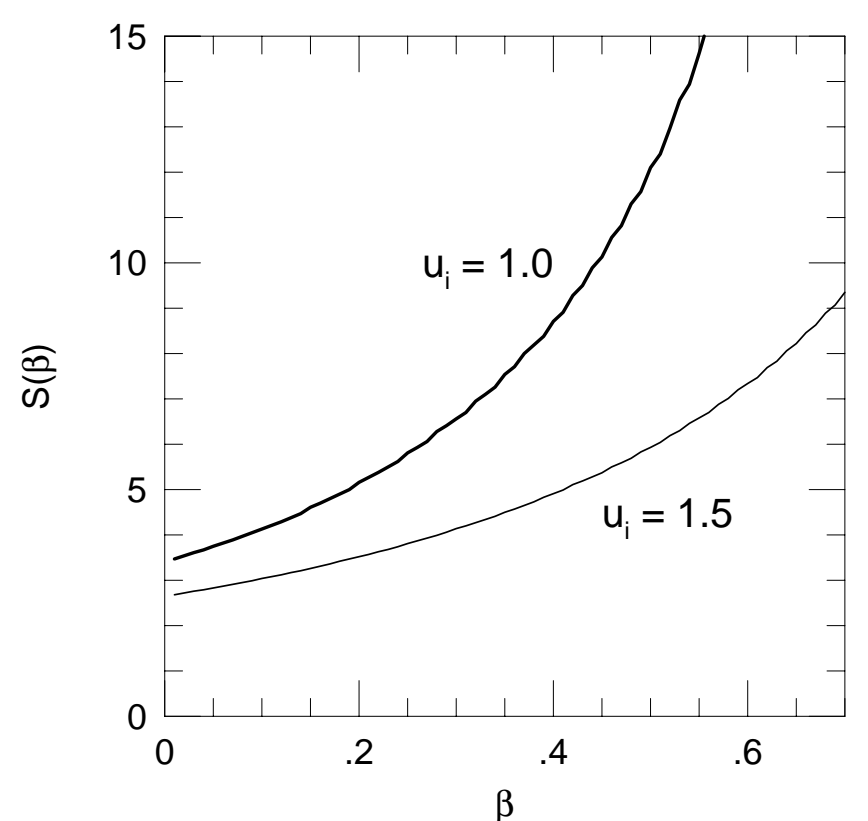

Fig. 2.- Normalized error, $S$, for the measurement of the asymmetry parameter, $\gamma$, plotted as a function of the impact parameter, $\beta$. The actual error is given by $\sigma_{\gamma}=S \sigma_{0}\left(N t_{\mathrm{E}} \mathrm{day}^{-1}\right)^{-1 / 2}$, where $\sigma_{0}$ is the factional flux error for an individual measurement and $N t_{\mathrm{E}} \mathrm{day}^{-1}$ is the number of measurements per Einstein crossing time. The heavy curve assumes that measurements begin when the source enters the Einstein ring $\left(u_{i}=1.0\right)$, and the solid curve assumes that they begin at $u_{i}=1.5$. In both cases, the observations are assumed to end at $t=t_{0}+1.5 t_{\mathrm{E}}$.

$\operatorname{ratio}(\mathrm{S} / \mathrm{N})$

$$
\begin{aligned}
\frac{\gamma}{\sigma_{\gamma}}= & 4.2 N^{1 / 2}\left(\frac{\sigma_{0}}{0.01}\right)^{-1}\left(\frac{S}{8}\right)^{-1}\left(\frac{\tilde{v}}{275 \mathrm{~km} \mathrm{~s}^{-1}}\right)^{-1} \\
& \times\left(\frac{t_{\mathrm{E}}}{40 \text { days }}\right)^{3 / 2} \frac{|\cos \phi|}{0.7}
\end{aligned}
$$

To detect the asymmetry from a typical halo event with $t_{\mathrm{E}} \sim 40$ days at the $4 \sigma$ level therefore requires about one observation per day, each with $\sigma_{0} \sim 1 \%$ accuracy. Assuming that $40 \%$ of the observing time is lost to the Moon and poor weather, this implies $\sim 1.7$ observations per clear and dark or gray night over the course of about 3 months.

For $t_{\mathrm{E}} \gtrsim \mathrm{yr} / 2 \pi$, equation (11) and the entire formalism underlying it break down because the parallax effect can no longer be represented as a simple asymmetry. That is, the two parallax parameters, $\tilde{v}$ and $\phi$, affect the light curve independently rather than through the single degenerate parameter $K_{\|}=\cos \phi / \tilde{v}$. Hence, much more information can be extracted from the event. By the same token, however, the description of the detectability of parallax becomes more complicated. For present purposes, we can obtain a lower limit on detectability by assuming that equation (11) saturates at $t_{\mathrm{E}} \sim \mathrm{yr} / 2 \pi$ and that all orientations $\phi$ are equally detectable (i.e., $\cos \phi \sim 1$ ). That is, for sufficiently long events the Earth-Sun axis is approximately aligned with $v$ for a duration about yr $/ 2 \pi \sim 58$ days. Therefore, for long events equation (11) should be replaced by

$$
\frac{\gamma}{\sigma_{\gamma}} \gtrsim 10 N^{1 / 2}\left(\frac{\sigma_{0}}{0.01}\right)^{-1}\left(\frac{S}{8}\right)^{-1}\left(\frac{\tilde{v}}{275 \mathrm{~km} \mathrm{~s}^{-1}}\right)^{-1}\left(2 \pi t_{\mathrm{E}} \gtrsim 1 \mathrm{yr}\right) \text {. }
$$




\section{REALISTIC OBSERVING REQUIREMENTS}

A $1 \mathrm{~m}$ telescope with reasonably good throughput detects $25 e \mathrm{~s}^{-1}$ from a $V=20$ star. (The use of broader filters is not advisable for reasons to be discussed below, and even standard filters may be too broad.) Thus, to obtain $1 \%$ photometry on a $V=20$ star requires a minimum exposure of $400 \mathrm{~s}$ on a $1 \mathrm{~m}$ telescope. If one assumes a mean sky of $V=21 \mathrm{mag} \operatorname{arcsec}^{-2}$ and $1^{\prime \prime}$ seeing, the exposure time is increased to $900 \mathrm{~s}$. The fields are generally crowded, which ordinarily would imply longer exposure times to acquire the same $\mathrm{S} / \mathrm{N}$. It is not completely clear, however, that substantially longer exposures would be required. According to equation (1), the parameters are estimated by first subtracting out a time-invariant background, $B$. This means that only pixel lensing (difference-image photometry) is required, not measurement of the absolute flux. Several groups are working on developing pixel-lensing techniques (Tomaney \& Crotts 1996; Ansari et al. 1997b; Melchior et al. 1998; Alard \& Lupton 1998; Tomaney 1998). While none have yet reached the photon limit on such deep exposures, several groups have come within a factor of 2 . For definiteness, I assume a $50 \%$ penalty in $\mathrm{S} / \mathrm{N}$ for crowding, add $60 \mathrm{~s}$ for overhead, and arrive at a total cycle time of $2100 \mathrm{~s}$. This means that $1 \mathrm{hr}$ per clear dark or gray night is required to follow each $V=20$ event.

The total number of events to be followed depends of course on the vigor of the microlensing search. To be concrete, I use as a model the first 2 yr of data from MACHO (Alcock et al. 1997a). A total of 12 events were, one way or another, selected as protocandidates. Many of these were not recognized until after the event had peaked, but I will assume that better triggers would have permitted all to be detected as they entered the Einstein ring. Events 2, 3, 11, and 12 were eventually rejected as candidates for various reasons; however, since events must be following beginning well before their peak, these protocandidates would had to have been monitored in any program designed to detect parallax asymmetries. Indeed, a number of other events would have probably been followed as well, at least for a while.

Of these 12 protocandidates, how many would yield parallax asymmetry measurements or useful upper limits? Only eight of the 12 were eventually deemed candidates. Of these, two of the sources (5 and 7) were $V \sim 21$ stars. The $\mathrm{S} / \mathrm{N}$ for these events would be a factor $\sim 2$ smaller than for the fiducial $V=20$ star calculated above and hence inadequate to detect an asymmetry unless the observation program was substantially more aggressive than the one envisaged above. Of course, this would have been recognized immediately, leading to a decision either to ignore these candidates or to focus more resources on them. I will assume the latter. Two other candidates (4 and 9) are nominally fairly bright $(V \sim 20$ and $V \sim 19.5)$ but this is only because they are blends. Both source stars are actually $V>21$. In this case, the faintness of the true source would not have been recognized until the follow-up was well underway. If this was recognized sufficiently early, more telescope resources could be applied to these events or they could have been dropped from the follow-up program. I will assume that these events would have been recognized as severe blends too late to acquire an adequate $S / N$. In summary, four $(1,6,8$, and 10) of the eight candidates would yield parallax measurements under the fiducial observing schedule, two (5 and 7) would yield parallaxes with 4 times the normal observing time, and the remaining two (4 and 9) would not produce useful information. Here I have not considered the timescales, binarity, or other known facts about the events in order to focus on the distributions of source brightness and blending.

For two of the events I allow 4 times longer for observation and therefore estimate that it would be necessary to monitor 12 events, each for an average of 90 minutes per clear dark or gray night over 3 months in order to make six useful measurements. This estimate may seem too conservative since, as I have indicated above, a number of the events would be dropped from the program prior to the full 3 months of observation. As also indicated above, however, an aggressive program would need to monitor some promising events that ultimately fail to be selected as candidates. Moreover, as I discuss in $\S 4$, each event may require additional observations beyond the 3 month interval in order to control various backgrounds. I therefore believe that this estimate of $50 \%$ efficiency is realistic.

To monitor six events per year would require an average of $2.25 \mathrm{hr}$ per night. This is of order one-quarter of the combined telescope time dedicated to follow-up of bulge microlensing events by the PLANET (Albrow et al. 1996, 1998) and GMAN (Alcock et al. 1996, 1997d) collaborations. I will therefore consider both what could be accomplished assuming that six events were followed per year and what could be done with a dedicated $1 \mathrm{~m}$ telescope (assuming that the initial search strategy were optimized to allow maximal follow-up). If the initial search could provide five events in each spring-summer period and three events in each fall-winter period, then a single $1 \mathrm{~m}$ telescope could devote approximately 90 minutes per night to each of them. These additional events would have to come from the outlying areas of the LMC. That is, a substantially larger area would have to be searched even during winter when conditions are unfavorable so as to provide events to monitor during the spring. MACHO actually monitored about twice as many stars as they analyzed in Alcock et al. (1997a), the remainder being in more outlying areas of the LMC. The EROS collaboration (Ansari 1997a) has recently begun a search over a still larger area employing a $1 \mathrm{deg}^{2}$ camera. Thus, one may expect an increase in the rate of candidates, especially if the lenses lie in the halo and not the LMC. The candidates from the outlying fields should be less crowded and thus easier to measure. However, for the same reason they should also be systematically fainter. For example, the EROS exposures in their outermost fields are $900 \mathrm{~s}$ compared to $180 \mathrm{~s}$ in their central fields. Thus it is not clear that the initial searches could provide fully 16 candidates per year that were bright enough for follow-up and on the required schedule. For definiteness, I estimate that 12 suitable candidates are found per year (i.e., double the current rate), one-half of which yield useful parallax asymmetry measurements. Thus, over 5 yr one could obtain about 15 useful measurements with one-quarter access to a $1 \mathrm{~m}$ telescope and 30 useful measurements with dedicated access.

A possible route to increasing the efficiency of the followup telescope would be to initiate the observations at $u_{i}=$ $1.5,\left[A\left(u_{i}\right)=1.13\right]$, rather than at $u_{i}=1$. This would be substantially more difficult and would risk more false detections, but, as Figure 2 shows, the improvement in $\mathrm{S} / \mathrm{N}$ would be dramatic, especially at higher impact parameters. 
One reason for this improvement is shown in Figure 1: for higher $\beta$, the first maximum deviation occurs at $t \lesssim t_{0}-t_{\mathrm{E}}$, which is missed if follow-up begins when the source enters the Einstein ring. A second reason is that for higher $\beta$, the peak time of the event $\left(t_{0}\right)$ is rather poorly determined if follow-up is delayed until the event enters the Einstein ring. The asymmetry parameter $\gamma$ is highly correlated with $t_{0}$ because both $\partial F / \partial t_{0}$ and $\partial F / \partial \gamma$ are odd functions of $\left(t-t_{0}\right)$. See equation (9).

\section{BACKGROUNDS}

The expected amplitude of the parallax asymmetry is only $\sim 1 \%$. There are several backgrounds, both astrophysical and geophysical, that might produce an asymmetry at this level. These backgrounds must be eliminated to the extent possible and accounted for in the analysis to the extent that they cannot be eliminated. I identify four such backgrounds.

First, the sources might be variable. It is straightforward to test various LMC populations for this level of variability simply by measuring the flux from other stars in the monitoring images. If $\sim 1 \%$ variability on $\sim 3$ month timescales is not extremely rare, then it will be necessary to continue to monitor the source after the end of the event to determine if it is such a variable. This is, of course, also straightforward but it does require substantial additional telescope time.

Second, differential refraction could cause a seasonal variation in the flux recovered from the source stars, since the stars will typically be observed at higher airmass in the winter than in the summer. If such an effect were present, it would produce a steady gradient in the recovered flux over the $\sim 3$ months between the times of maximum expected asymmetrical deviation $\left(t \sim t_{0} \pm t_{\mathrm{E}}\right)$ and thus give the impression of an asymmetry when there was none. The recovered flux would change as differential refraction pushed background stars of different color closer to (or farther from) the source star. Thus, this effect could mimic asymmetries of either sign. Again, it would be straightforward to test for the general presence of such an effect by monitoring other stars in the field. If $\sim 1 \%$ amplitudes were not rare, one could as before measure the size of the effect for the source star by continuing the monitoring after the event was over. Again, the only cost would be telescope time. It would be possible to eliminate this effect altogether by using sufficiently narrow filters; however, in view of the severe $\mathrm{S} / \mathrm{N}$ requirements, this approach would be selfdefeating. Since the effect worsens with the width of the filters, very wide filters are probably not a good route to higher $\mathbf{S} / \mathbf{N}$.

Third, binary source events could be asymmetric (Griest \& $\mathrm{Hu} 1992)$ and therefore could mimic parallax asymmetries. Consider, for example, a binary whose primary is lensed and whose secondary is $15 \%$ as bright as the primary. The secondary lies at $\Delta \boldsymbol{u}=(1,1)$ where the first component represents the separation along the direction of motion relative to the lens, and the second component represents the separation along the perpendicular direction. Suppose that the impact parameter of the event was $\beta=0.4$ and the lens lay on the opposite side of the primary source from its companion. Then in units of the primary plus secondary flux, the total magnified flux would be 1.259 at $t=t_{0}-t_{\mathrm{E}}$ and 1.275 at $t=t_{0}+t_{\mathrm{E}}$. That is, the binary would mimic a light curve with an asymmetry of $1.2 \%$. Of course, the detailed structure of the binary-source light curve would be quite different from one produced by parallax asymmetry, but since according to equation (11) the asymmetry is only just barely being detected, in most cases one could not distinguish between the two.

What fraction of events will have asymmetries owing to binaries? Only binaries with magnitude differences $\Delta V \lesssim 4$ and therefore mass ratios $q \gtrsim 0.45$ (Henry \& McCarthy 1993) can contribute. Other companions contribute too little flux to make a detectable asymmetry. Griest \& $\mathrm{Hu}$ (1992) have compiled data on binaries from various sources and report that $\sim 40 \%$ of $F$ stars and $\sim 60 \%$ of $A$ stars satisfy this criterion. Similarly, only binaries with sourcelens separations $\Delta \boldsymbol{u} \lesssim 3$ can contribute, since beyond this radius the flux from the secondary is nearly constant with time. About half of the binaries within this radius, however, generate light curves that differ substantially from parallax asymmetries. For example, if the binary is aligned along the $y$-axis, $\Delta \boldsymbol{u}=\left(0, \Delta u_{y}\right)$, then the light curve distortion will be symmetric. If it lies along the $x$-axis, the distortion will be double peaked. The fraction of binaries satisfying $\Delta u \lesssim 3$ depends on what physical separation corresponds to an Einstein radius. As a practical matter, however, the dependence is rather weak. If for example the lenses are $0.5 M_{\odot}$ stars in the LMC, with lens-source separations $d_{1 \mathrm{~s}}=3 \mathrm{kpc}$, then an Einstein radius corresponds to $\left(d_{\mathrm{os}} / d_{\mathrm{ol}}\right) r_{\mathrm{E}} \sim 3.5 \mathrm{AU}$. If they are $0.5 M_{\odot}$ objects in the halo with $d_{\mathrm{ol}} \sim 10 \mathrm{kpc}$, then $\left(d_{\mathrm{os}} / d_{\mathrm{ol}}\right) r_{\mathrm{E}} \sim 28 \mathrm{AU}$. According to the table assembled by Griest \& Hu (1992), $32 \%$ of F stars and $42 \%$ of A stars have separations within three Einstein radii in the first case, and $41 \%$ of $F$ stars and $47 \%$ of $A$ stars do so in the second case. I take the average of these two cases and assume that two-thirds of all $\mathrm{A}$ and $\mathrm{F}$ stars have binary companions. I then estimate $5 \%$ of $\mathrm{F}$ stars and $9 \%$ of A stars will have companions that generate asymmetries that could be mistaken for parallax effects. This fraction is small but not negligible.

The last effect that can mimic parallax asymmetries is a binary lens of projected separation, $b$. If $b / r_{\mathrm{E}} \sim 1$, then the light curve generally has caustics or other features that easily distinguish it from a standard microlensing light curve. However, for $0.25 \lesssim b / r_{\mathrm{E}} \lesssim 0.6$ and for $1.4 \lesssim b / r_{\mathrm{E}} \lesssim$ 3 , binary-lens light curves can look similar to standard light curves except that they have a slight asymmetry. For $b \lesssim$ $0.25 r_{\mathrm{E}}$ the binary acts effectively as a point lens (Gaudi \& Gould 1997b), and for $b \gtrsim 3 r_{\mathrm{E}}$, the effect of the companion is either too small to be noticed or causes a double-peaked rather than an asymmetric event. It is more difficult to estimate a priori what is the level of contamination from binary lenses than from binary sources because unlike the source population, the lens population is almost completely unknown; however, it is possible to estimate the level of contamination from the microlensing data themselves.

Both the angle $\theta$ between $v$ and the binary separation vector and the impact parameter $\beta$ are randomly distributed. At all binary separations there are some combinations of $\theta$ and $\beta$ that give rise to easily recognizable effects. For example, for separations $b \sim 2 r_{\mathrm{E}}$ and $\theta=0$, the events will be double peaked. For $b \lesssim 0.6 r_{\mathrm{E}}$, the character of the recognizable deviations is more difficult to describe but can be gauged from Figure 1 of Gaudi \& Gould (1997b). Thus, in principle one can estimate the binary fraction and the binary-separation and mass-ratio distributions by cataloging these recognizable events and determining the efficiency of their detection by Monte Carlo simulation. Once 
this distribution is known, one can predict the number of asymmetric events that are indistinguishable from parallax, again by Monte Carlo.

In practice, the precision of these estimates will be limited by the small number of events that have clear characteristics of binary lenses. Since the rate of such clear cases is of order the rate of asymmetric binary-lens events, however, this procedure should produce an estimate of the background that is only somewhat larger than the Poisson error.

At present, it is possible to give only a very rough estimate of the contamination by binary lenses. I do so as follows. I note that one of the eight MACHO LMC candidates is clearly a binary. I assume that the number of contaminants is approximately equal to the number of clear-cut binaries and therefore estimate a contamination rate of $12 \%$. Hence the overall contamination rate from binary lenses and binary sources is about $19 \%$.

\section{RESOLVING THE NATURE OF THE LENSES}

What could be accomplished with a 5 yr follow-up program? I consider first the more modest scenario with one-quarter access to a $1 \mathrm{~m}$ telescope that, as discussed in $\S 3$, could be expected to yield 15 useful measurements. Let us suppose that most (say $80 \%$ ) of the lenses reside in the halo and the rest are in the Galactic disk or the LMC. Of the expected 12 halo events, only about two-thirds would have measurable parallaxes. For the remainder, the combination $|\cos \phi| / S$ would fall substantially below the value $\sim 0.09$ assumed in equation (11). Thus, one would expect eight detectable events plus 1.3 background events among the remaining seven events with undetectable parallaxes (see $\S 4$ ). (Insofar as there were Galactic-disk events, these would also be detectable, but because of their large parallaxes would usually be recognizable as such.) On the other hand, if there were no halo lenses one would still expect about one additional spheroid event that would mimic the parallax characteristics of a halo lens plus 2.7 background events. Assuming that the variance of the background is twice Poisson, these two scenarios would therefore imply
$9.3 \pm 2.2$ and $3.7 \pm 2.3$ asymmetric events, respectively. These two ranges are only marginally separated. If there were, for example, three or 10 asymmetry detections, then the results would be unambiguous. If there were seven detections, however, the situation would be less clear.

With a dedicated $1 \mathrm{~m}$ telescope, the two scenarios would imply $18.6 \pm 3.1$ and $7.4 \pm 3.3$ asymmetric events. These ranges are well separated and one could unambiguously discriminate between them.

Even the more aggressive version of this experiment, however, would leave a number of questions unresolved. For example, if a large fraction of the events had measurable asymmetries, one could still not tell whether they lay in a halo or in the Evans et al. (1998) flared disk. Moreover, because the measurable quantity $K_{\|}=\cos \phi / \tilde{v}$ depends on the unknown angle $\phi$, the velocity dispersion of a detected halo would be poorly constrained. One therefore could not distinguish between a "true" $\left(r^{-2}\right)$ halo and a heavy spheroid $\left(r^{-3.5}\right)$ whose velocity dispersion would be expected to be smaller by a factor of $(2 / 3.5)^{1 / 2} \sim 0.75$ (assuming a fixed logarithmic potential). Likewise, one could not reliably detect rotation of the halo. These additional pieces of information, which are crucial to understanding the formation process and therefore the nature of the halo lenses (if that is what they are), could be obtained from satellite parallaxes but not from the ground.

Finally, I note that of the two bulge follow-up collaborations, the PLANET telescope time is largely concentrated in the bulge season, but GMAN has access to significant telescope time on a year-round basis. GMAN has in fact followed several LMC events although the results have not yet been published. It therefore should be possible to initiate at least a modest version of the observing program outlined here on the basis of currently available telescope resources.

I would like to thank Piotr Popowski for a careful reading of the manuscript. This work was supported in part by grant AST 94-20746 from the NSF and in part by grant NAG 5-3111 from NASA.

\section{REFERENCES}

Alard, C., \& Lupton, R. H. 1998, ApJ, 503, 325

Albrow, M., et al. 1996, in IAU Symp. 173, Astrophysical Applications of Gravitational Microlensing, ed. C. S. Kochanek \& J. N. Hewitt (Dordrecht: Kluwer), 227

. 1998, ApJ, in press

Alcock, C., et al. 1995, ApJ, 454, L125 1996, ApJ, 463, L67 $1997 \mathrm{a}$, ApJ, 486, 697 1997b, ApJ, 490, 59 1997c, ApJ, 491, L11 1997d, ApJ, 491, 436

Ansari, R., et al. 1997a, A\&A, 324, 69 1997b, A\&A, 324, 843

Beaulieu, J.-P., \& Sackett, P. D. 1998, AJ, 116, 209

Bennett, D. 1998, ApJ, 493, L79

Bennett, D., et al. 1997, BAAS, 191, 8308

Boutreux, T., \& Gould, A. 1996, ApJ, 462, 705

Buchalter, A., \& Kamionkowski, M. 1997, 482, 782

Evans, N. W., Gyuk, G., Turner, M. S., \& Binney, J. J. 1998, ApJ, 501, L29

Gaudi, B. S., \& Gould, A. 1997a, ApJ, 477, 152

.1997b, ApJ, 482, 83
Gould, A. 1992, ApJ, 392, 442

1994, ApJ, 421, L75

1995a, ApJ, 441, L21

$1995 b$, ApJ, 441, 77

1998, ApJ, 499, 728

Gould, A., Miralda-Escudé, \& Bahcall, J. N. 1994, ApJ, 423, L105

Gould, A., \& Welch, D. L. 1996, ApJ, 464, 212

Griest, K., \& Hu, W. 1992, ApJ, 397, 362

Han, C., \& Gould, A. 1997, ApJ, 480, 196

Henry, T. J., \& McCarthy, D. W. 1993, AJ, 106, 773

Marković, D. 1998, MNRAS, in press

Melchior, A.-L. 1998, A\&A, in press

Paczyński, B. 1986, ApJ, 304, 1

Palanque-Delabrouille, N., et al. 1998, A\&A, 332, 1

Refsdal, S. 1966, MNRAS, 134, 315

Sahu, K. C. 1994, Nature, 370, 275

Tomaney, A. 1998, preprint (astro-ph/9801233)

Tomaney, A., \& Crotts, A. P. S. 1996, AJ, 112, 2872

Wu, X.-P. 1994, ApJ, 435, 66

Zaritsky, D., \& Lin, D. N. C. 1997, AJ, 114, 254

Zhao, H. 1998, MNRAS, 294, 139 